\title{
On the Velocity Gradient in Stably Stratified Sheared Flows. Part 1: Asymptotic Analysis and Applications
}

\author{
S. S. Zilitinkevich · I. Esau • N. Kleeorin • \\ I. Rogachevskii · R. D. Kouznetsov
}

Received: 11 August 2009 / Accepted: 9 March 2010 / Published online: 27 March 2010

(C) The Author(s) 2010. This article is published with open access at Springerlink.com

\begin{abstract}
We give a new derivation of the familiar linear relation for the dimensionless velocity gradient in the stably stratified surface layer and provide physical and empirical grounds for its universal applicability in stationary homogeneous turbulence over the whole range of static stabilities from $R i=0$ to very large $R i$. Combining this relation with the budget equation for the turbulent kinetic energy we obtain the "equilibrium formulation" of the turbulent dissipation length scale, and recommend it for use in turbulence closure models.
\end{abstract}

Keywords Flux Richardson number $\cdot$ Stationary and homogeneous regime $\cdot$ Strong static stability · Turbulence closure - Turbulent dissipation length scale · Turbulent kinetic energy · Velocity gradient

\section{Traditional Similarity Theory Formulation for the Velocity Gradient}

The key point of our paper is to provide new insight into the familiar relation for the velocity gradient in the stably stratified atmospheric surface layer:

S. S. Zilitinkevich $(\varangle) \cdot$ R. D. Kouznetsov

Finnish Meteorological Institute, Helsinki, Finland

e-mail: sergej.zilitinkevich@fmi.fi

S. S. Zilitinkevich · I. Esau

G.C. Rieber Climate Institute, Nansen Environmental and Remote Sensing Centre, Bergen, Norway

S. S. Zilitinkevich · R. D. Kouznetsov

A.M. Obukhov Institute of Atmospheric Physics, Moscow, Russia

S. S. Zilitinkevich

Department of Physics, University of Helsinki, Helsinki, Finland

I. Esau

Bjerknes Centre for Climate Research, Bergen, Norway

N. Kleeorin · I. Rogachevskii

Department of Mechanical Engineering, Ben-Gurion University of the Negev, Beer-Sheva, Israel 


$$
\frac{\partial U}{\partial z}=\frac{|\tau|^{1 / 2}}{k z}\left(1+C_{u} \frac{z}{L}\right)
$$

where $L$ is the Obukhov length ${ }^{1}$ :

$$
L \equiv \frac{|\tau|^{3 / 2}}{-\beta F_{\theta}},
$$

$z$ is the height, $U$ is the mean wind velocity, $\tau=\overline{u w}$ and $F_{\theta}=\overline{\theta w}$ are vertical turbulent fluxes of momentum and potential temperature, $u$ and $w$ are fluctuations of horizontal (along the mean wind) and vertical velocities, $\theta$ is the fluctuation of potential temperature, the overbar denotes statistical averaging, $\beta=g / T_{0}$ is the buoyancy parameter, $g$ is the acceleration of gravity, $T_{0}$ is a reference value of the absolute temperature $(\Theta), k \approx 0.4$ (the von Karman constant) and $C_{u} \approx 2$ are empirical dimensionless constants.

Equation 1 has been derived from the following concept of " $z$-less stratification": basic properties of turbulence in stable stratification depend on $z$ close to the surface, but this dependence decreases with increasing $z$ and sufficiently far from the surface, at $z>>L$, becomes negligible. If so, simple dimensional analysis yields the following asymptotic relations for the eddy viscosity:

$$
K_{M} \sim|\tau|^{1 / 2} L
$$

and the velocity gradient:

$$
\frac{\partial U}{\partial z}=\frac{-\tau}{K_{M}} \sim \frac{|\tau|^{1 / 2}}{L} .
$$

Then Eq. 1 is immediately derived as the simplest interpolation between the classical wall law:

$$
\frac{\partial U}{\partial z}=\frac{|\tau|^{1 / 2}}{k z}
$$

and Eq. $4 \mathrm{a}$ in the form

$$
\frac{\partial U}{\partial z}=\frac{C_{u}}{k} \frac{|\tau|^{1 / 2}}{L} .
$$

Data from observations in the atmospheric surface layer for $0<z / L<10$ generally confirm Eq. 1 with a reasonably certain value of $C_{u} \approx 2$ (e.g., Dyer 1974; Högström 1996; Zilitinkevich and Esau 2007).

\section{Alternative Derivation}

We recall that the turbulent regime corresponding to $z / L>1$ is often considered in boundary-layer meteorology as "strongly stable". This wording is not quite accurate. In a more general context, the static stability is characterised (alternatively to $z / L$ ) by the gradient Richardson number:

$$
R i \equiv \frac{\beta \partial \Theta / \partial z}{(\partial U / \partial z)^{2}}
$$

\footnotetext{
${ }^{1}$ Recall that the original definition of the Obukhov length included the von Karman constant in the denominator. Using this definition, the numerical coefficient analogous to $C_{u}=2$ in Eq. 1 would be $C_{u} / k=5$.
} 
Typical values of $R i$ in the atmospheric stable planetary boundary layer (PBL) do not exceed the threshold $R i=0.25$ (e.g. Garratt 1992), whereas the free atmosphere is characterised by $R i$ of order 10 . Furthermore, it seems reasonable to define the upper boundary of the stable PBL just as the height at which $R i$ passes the above threshold (Zilitinkevich et al. 2008). From this point of view, the stable PBL flow should be considered as weakly stable, so that Eqs. 4a and 4b represent so called "intermediate asymptotes" (see Barenblatt 1996).

To answer the question as to whether Eqs. $4 \mathrm{a}$ and $4 \mathrm{~b}$ are applicable or not in really strong static stability (that is $R i>>1$ ), we consider the familiar budget equation for the turbulent kinetic energy (TKE), $E_{K}=\frac{1}{2}\left(\overline{u^{2}}+\overline{v^{2}}+\overline{w^{2}}\right)$, in the stationary homogeneous turbulent regime:

$$
\left(\frac{d E_{K}}{d t}=\right)-\tau \frac{\partial U}{\partial z}+\beta F_{\theta}-\varepsilon_{K}=0,
$$

where $-\tau \partial U / \partial z>0$ is the TKE production by the wind shear, $\beta F_{\theta}<0$ is the TKE consumption for overtaking the buoyancy forces, and $\varepsilon_{K}>0$ is the TKE viscous dissipation rate. In this regime, the absolute value of the buoyancy flux, $\beta F_{\theta}$, could only increase ${ }^{2}$ with strengthening stability (that is with increasing $R i$ ); but, by virtue of Eq. 6, it cannot exceed $-\tau \partial U / \partial z$. Then the flux Richardson number:

$$
R i_{f} \equiv \frac{-\beta F_{\theta}}{-\tau \partial U / \partial z}
$$

is a monotonically increasing but limited function of $R i$ or $z / L$ (see Fig. 1). Therefore at $R i \rightarrow \infty$ it should tend to a finite limit:

$$
\frac{-\beta F_{\theta}}{-\tau \partial U / \partial z} \rightarrow R_{\infty}=\text { constant }<1 .
$$

Equation 8 yields the large- $R i$ asymptotes: a linear relation for the turbulent Prandtl number:

$$
\operatorname{Pr}_{T} \equiv \frac{K_{M}}{K_{H}}=\frac{\tau(\partial \Theta / \partial z)}{F_{\theta}(\partial U / \partial z)} \rightarrow \frac{R i}{R_{\infty}}
$$

where $K_{M}$ and $K_{H}$ are the eddy viscosity and eddy conductivity; and the same relation as Eq. $4 \mathrm{a}$ for the velocity gradient:

$$
\frac{\partial U}{\partial z} \rightarrow \frac{-\beta F_{\theta}}{-R_{\infty} \tau}=\frac{|\tau|^{1 / 2}}{R_{\infty} L} .
$$

The maximum value of the flux Richardson number: $R_{\infty} \approx 0.2$ has been determined from various experimental, large-eddy simulation (LES) and direct numerical simulation (DNS) data for extremely strong static stabilities, namely, for the values of Ri between 1 and $10^{2}$, at which $R i_{f}$ definitely levels off (e.g., Yamada 1975; Stroscio 1982; Zilitinkevich et al. 2007, 2008, 2009; Mauritsen and Svensson 2007; Mauritsen et al. 2007; Stretch et al. 2009; Venayagamoorthy and Stretch 2010).

It is remarkable that the coefficient $1 / R_{\infty} \approx 5$ in Eq. 10 based on data for extremely stable stratification ( $R i$ up to $10^{2}$ ) coincides with the coefficient $C_{u} / k \approx 5$ in Eq. $4 \mathrm{c}$ based on experimental and LES data for the atmospheric surface layer (that is the lower part of

\footnotetext{
${ }^{2}$ In the stationary homogeneous regime the static stability is equivalently characterised by $R i, z / L$ or $R i_{f}$. However, this is not generally true in non-homogeneous or non-stationary turbulence. At large $R i$, the heat flux could decrease with increasing stability (e.g., Basu et al. 2006); cf. examples of non-monotonic dependence of $R i_{f}$ on $R i$ in Mahalov et al. (2004) and a detailed discussion of atmospheric data by Kouznetsov and Zilitinkevich (2010).
} 

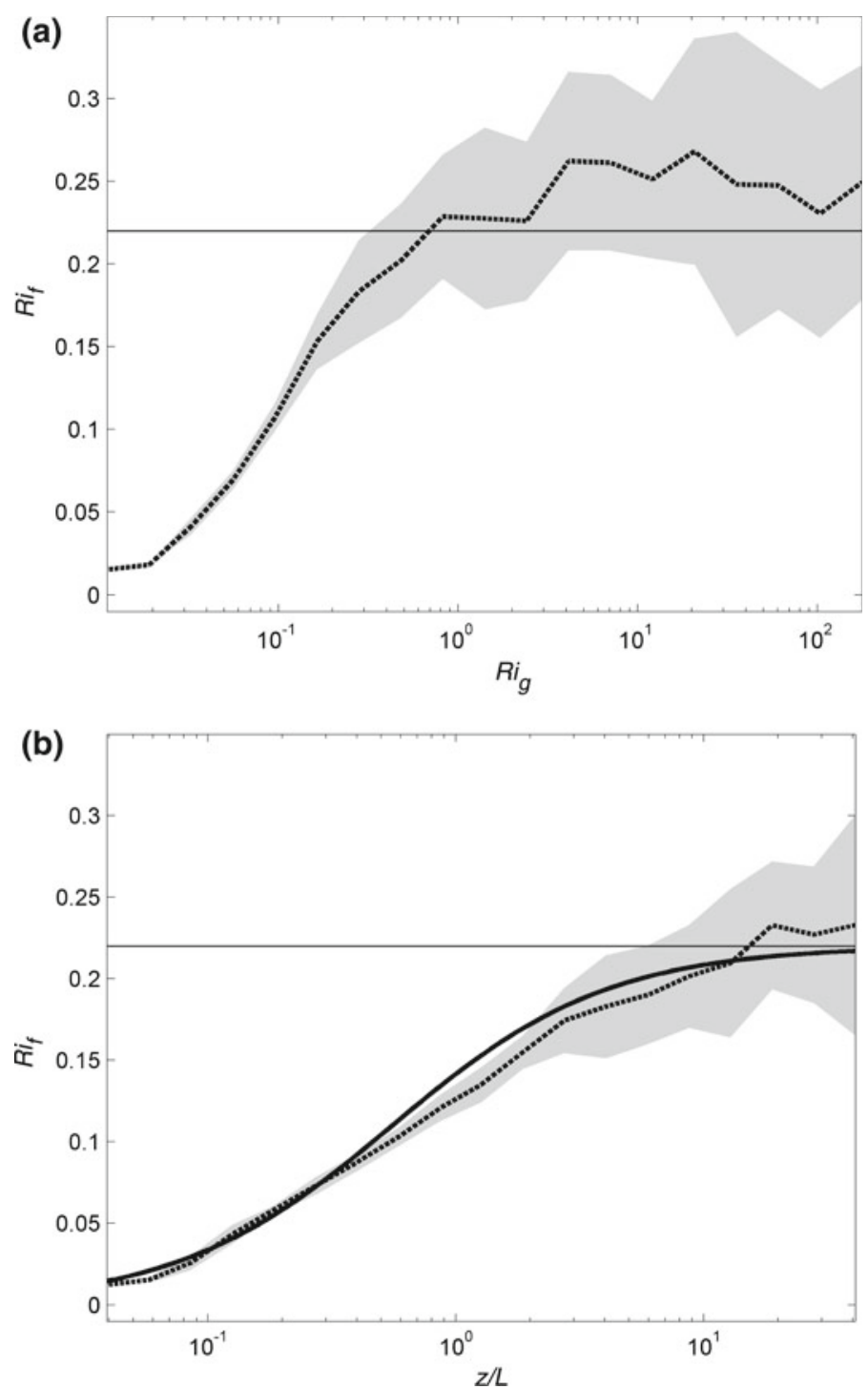

Fig. 1 The stability dependence of the flux Richardson number after LES DATABASE64 (Esau and Zilitinkevich 2006) supplemented with several high-resolution runs: a $R i_{f}$ versus $R i$; b $R i_{f}$ versus $z / L$, with the theoretical curve (solid line) plotted after Eq. 16 with $k=0.4$ and $R_{\infty}=0.22$. Dashed lines show averaged empirical data; grey area is three standard deviations

the boundary layer, where Richardson numbers do not exceed 0.25 ). In other words, the intermediate, "surface-layer asymptote" Eq. 4c (relevant to large $z / L$ but still quite small $R i$ ) coincides with the ultimate "very large $R i$ asymptote" (relevant to the extremely stable homogeneous turbulence that is unapproachable in boundary-layer flows). It follows that Eq. 1 is applicable to stationary and homogeneous stably stratified flows over the entire range of static stabilities from neutral to extremely stable; whereby $C_{u}$ is expressed through the independently determined fundamental empirical constants: 


$$
C_{u}=k / R_{\infty}
$$

In this statement the words "stationary and homogeneous" should be emphasised. It goes without saying that the velocity gradients in stable stratification, in particular in the upper part of the stable PBL, are often inconsistent with Eq. 1. Kouznetsov and Zilitinkevich (2010) have demonstrated that such inconsistencies are always associated with flow heterogeneity and/or the non-stationary evolution of the flow.

\section{Turbulent Dissipation Length Scale}

Following Kolmogorov (1941), the TKE dissipation rate, $\varepsilon_{K}$, is expressed through the dissipation time scale $t_{T}$ or the integral turbulent length scale $l \equiv E_{K}^{1 / 2} t_{T}$ :

$$
\varepsilon_{K}=\frac{E_{K}}{C_{K} t_{T}}=\frac{E_{K}^{3 / 2}}{C_{K} l},
$$

where $C_{K}$ is a dimensionless universal constant. It is worth emphasising that Eq. 12 does not imply any hypotheses: it merely substitutes the unknown $\varepsilon_{K}$ by the alternative unknowns, $t_{T}$ or $l$. Determination of $l$ is therefore one of the key aspects of the general turbulence closure problem.

In a neutrally stratified, plain-parallel, non-rotating boundary-layer flow the maximum sizes of locally generated turbulent eddies are limited only by the distance, $z$, from the underlying surface. Then the basic neutral-stability turbulent length scale, $l=l_{0}$, can be taken as proportional to $z$. Identifying the proportionality constant with the free constant $C_{K}$ in Eq. 12, we can take

$$
l_{0}=z .
$$

Equations 6, 12 and 13 yield the relation $\partial U / \partial z=\tau^{1 / 2} /\left[C_{K}\left(\tau / E_{K}\right)^{3 / 2} z\right]$ consistent with the classical wall law: $\partial U / \partial z=\tau^{1 / 2} / k z$, where

$$
k=C_{K}\left(\frac{|\tau|}{E_{K}}\right)_{R i=0}^{3 / 2}
$$

is the von Karman constant.

Lo et al. (2005) derived a quite similar relationship between $k$ and $\left(\tau / E_{K}\right)_{R i=0}$ for stationary and homogenous sheared flows: $k=C_{2}^{3 / 2}\left(\tau / E_{K}\right)_{R i=0}$ and determined the constant $C_{2} \approx 2$ (that appears in the second-order velocity structure function) from a large number of laboratory and numerical experiments. Relying on this estimate, we obtain $C_{K}=C_{2}^{3 / 2} \approx 2.8$. For comparison, taking $\left(\tau / E_{K}\right)_{R i=0} \approx 0.3$ (after Zilitinkevich et al. 2008) and $k \approx 0.4$, Eq. 14 yields a reasonably close value of $C_{K} \approx 2.4$. Closure models based on the steady or non-steady versions of the TKE budget equation with $\varepsilon_{K}$ parameterized through Eqs. 12, 13 reasonably accurately reproduce neutrally stratified turbulent flows. In stable stratification, physical mechanisms controlling turbulence are much more complicated and the turbulent length scale in closure models is still determined heuristically and rather uncertainly.

In view of analyses given in Sect. 2, Eq. 1 with $C_{u}=k / R_{\infty}$ can be considered as an inherent property of stationary homogeneous turbulence, which allows us to determine $l$ from Eqs. 1, 6 and 12:

$$
l=\frac{k}{C_{K}}\left(\frac{E_{K}}{|\tau|}\right)^{3 / 2} \frac{z}{1+k\left(R_{\infty}^{-1}-1\right) z / L}
$$


It is worth noting that Eq. 15 does not imply a simple linear interpolation between $z^{-1}$ and $L^{-1}$, because the ratio $E_{K} /|\tau|$ essentially depends on the static stability.

Substituting Eq. 1 with $C_{u}=k / R_{\infty}$ into Eq. 7 yields a simple relation linking $z / L$ and $R i_{f}$ :

$$
\frac{z}{L}=\frac{R_{\infty}}{k} \frac{R i_{f}}{R_{\infty}-R i_{f}},
$$

so that $l$ can also be expressed in terms of the neutral-stability turbulent length scale $l_{0}$ (equal to $z$ in boundary-layer flows) and the flux Richardson number:

$$
l=\frac{k l_{0}}{C_{K} R_{\infty}}\left(\frac{E_{K}}{-\tau}\right)^{3 / 2} \frac{R_{\infty}-R i_{f}}{1-R i_{f}} .
$$

The stability dependence of $E_{K} /|\tau|$ has been examined theoretically and empirically by Zilitinkevich et al. (2007, 2008, 2009), Mauritsen and Svensson (2007) and Mauritsen et al. (2007). With increasing stability, $E_{K} /|\tau|$ increases starting from 3 to 5 in neutral stratification (Mann 1994; Lo et al. 2005) and approaches 10-15 in very strong static stability at $R i>10$. Then, as follows from Eq. 15, in the stability range $0<z / L<5$ typical of the atmospheric boundary layer, $l$ should remain weakly sensitive to the stratification and basically proportional to $z$; whereas the strong stability limit, $l \sim L$, is approached only in the free atmosphere, outside the boundary layer. This explains why old attempts to determine $l$ through simple interpolation: $1 / l=1 / z+$ (constant) $/ L$ happened to be a poor approximation (see Heinemann 2004; van de Wiel et al. 2008).

\section{Concluding Remarks}

Asymptotic analysis of the stationary, homogeneous TKE budget equation provides a principal applicability of the surface-layer velocity gradient formulation, Eq. 1, up to extremely strong static stabilities, and allows expressing the empirical constant $C_{u}$ in Eq. 1 through the von Karman constant $k \approx 0.4$ and the maximal flux Richardson number $R_{\infty} \approx 0.2$. On this basis we determine the stability dependence of the turbulent length scale in terms of the dimensionless height $z / L$ (Eq. 15) or flux Richardson number $R i_{f}$ (Eq. 17), and recommend this formulation for use in turbulence closure models.

We emphasise that the above formulations are valid only in the stationary, homogeneous regime. In part II of our study, Kouznetsov and Zilitinkevich (2010) analyse examples of the violation of Eq. 1 known from the literature, and attribute them to the essential heterogeneity of turbulence in the upper parts of a stable boundary layer.

Acknowledgements This work has been supported by the EC FP7 projects ERC PBL-PMES (No. 227915) and MEGAPOLI (No. 212520); and the Norwegian Research Council project 191516/V30 Planetary Boundary Layer Feedback in the Earth's Climate System.

Open Access This article is distributed under the terms of the Creative Commons Attribution Noncommercial License which permits any noncommercial use, distribution, and reproduction in any medium, provided the original author(s) and source are credited. 


\section{References}

Barenblatt GI (1996) Scaling, self-similarity, and intermediate asymptotics. Series: Cambridge texts in applied mathematics, vol 14. Cambridge University Press, Cambridge, 386 pp. ISBN 05214-35226

Basu S, Porté-Agel F, Foufoula-Georgiou E, Vinuesa JF, Pahlow M (2006) Revisiting the local scaling hypothesis in stably stratified atmospheric boundary-layer turbulence: an integtration of field and laboratory measurements with Large-Eddy simulations. Boundary-Layer Meteorol 119:473-500. doi:10.1007/ s10546-005-9036-2

Dyer AJ (1974) A review of flux-profile relationships. Boundary-Layer Meteorol 7:363-372

Esau IN, Zilitinkevich SS (2006) Universal dependences between turbulent and mean flow parameters in stably and neutrally stratified planetary boundary layers. Nonlinear Processes Geophys 13:135-144

Garratt JR (1992) The atmospheric boundary layer. Cambridge atmospheric and space science series. Cambridge University Press, Cambridge, $316 \mathrm{pp}$

Heinemann G (2004) Local similarity properties of the continuously turbulent stable boundary layer over Greenland. Boundary-Layer Meteorol 112:283-305

Högström U (1996) Review of some basic characteristics of the atmospheric surface layer. Boundary-Layer Meteorol 78:215-246

Kolmogorov AN (1941) Energy dissipation in locally isotropic turbulence. Dokl Akad Nauk SSSR 32(1): $19-21$

Kouznetsov RD, Zilitinkevich SS (2010) On the velocity gradient in the stably stratified sheared flows. Part 2: observations and models. Boundary-Layer Meteorol. doi:10.1007/s10546-010-9487-y

Lo TS, L'vov VS, Pomyalov A, Procaccia I (2005) Estimating von-Karman's constant from homogeneous turbulence. http://arxiv.org/abs/nlin/0506044v1

Mahalov A, Nicolaenko B, Tse KL, Joseph B (2004) Eddy mixing in jet-stream turbulence under stronger stratification. Geophys Res Lett 31:L23111. doi:10.1029/2004GL021055

Mann J (1994) The special structure of neutral atmospheric surface layer turbulence. J Fluid Mech 273: $141-168$

Mauritsen T, Svensson G (2007) Observations of stably stratified shear-driven atmospheric turbulence at low and high Richardson numbers. J Atmos Sci 64:645-655

Mauritsen T, Svensson G, Zilitinkevich SS, Esau I, Enger L, Grisogono B (2007) A total turbulent energy closure model for neutrally and stably stratified atmospheric boundary layers. J Atmos Sci 64:4117-4130

Stretch DD, Rottman JW, Venayagamoorthy SK, Nomura KK, Rehmann CR (2009) Mixung efficiency in decaying stably stratified turbulence. Dyn Atmos Oceans. doi:10.1016/j.dynatmoce.2008.11.002

Stroscio MA (1982) Enhancement of turbulence in a stratified fluid by the presence of a shear field. J Stat Phys 28(3):607-612

van de Wiel BJH, Moene AF, De Ronde WH, Jonker HJJ (2008) Local similarity in the stable boundary layer and mixing-length approaches: consistency of concepts. Boundary-Layer Meteorol 128:103-116

Venayagamoorthy SK, Stretch DD (2010) On the turbulent Prandtl number in homogeneous stably stratified turbulence. J Fluid Mech 644:359-369

Yamada T (1975) The critical Richardson number and the ratio of the Eddy transport coefficients obtained from a turbulence closure model. J Atmos Sci 32:926-933

Zilitinkevich S, Esau I (2007) Similarity theory and calculation of turbulent fluxes at the surface for the stably stratified atmospheric boundary layers. Boundary-Layer Meteorol 125:193-296

Zilitinkevich SS, Elperin T, Kleeorin N, Rogachevskii I (2007) Energy- and flux-budget (EFB) turbulence closure model for the stably stratified flows. Part I: Steady-state, homogeneous regimes. Boundary-Layer Meteorol 125:167-192

Zilitinkevich SS, Elperin T, Kleeorin N, Rogachevskii I, Esau I, Mauritsen T, Miles MW (2008) Turbulence energetics in stably stratified geophysical flows: strong and weak mixing regimes. Q J R Meterol Soc 134:793-799

Zilitinkevich SS, Elperin T, Kleeorin N, L'vov V, Rogachevskii I (2009) Energy- and flux-budget (EFB) turbulence closure model for stably stratified flows. Part II: The role of internal gravity waves. Boundary-Layer Meteorol 133:139-164 